\title{
Overcoming Intrinsic and Coupling Spin Resonances in the AGS $^{1}$
}

\author{
M. Bai, L. Ahrens, T. Roser \\ Brookhaven National Laboratory, Upton, NY 11973, U.S.A
}

\begin{abstract}
In the Brookhaven AGS, polarized protons are accelerated from $G \gamma=4.5$ to $G \gamma=46.5$. During the acceleration, a total of 42 imperfection spin depolarization resonances and 7 intrinsic spin resonances are crossed. Currently, the depolarization at each imperfection spin resonance is overcome by a solenoid $5 \%$ snake and full spin flips are induced at 4 out of the 7 intrinsic resonances by the AGS rf dipole to avoid the polarization loss. No correction schemes are applied at the remaining 3 weak spin resonances. In addition, coupling spin resonances are also observed due to the solenoidal field of the snake and no correction is applied for these spin resonances other than keeping the horizontal and vertical betatron tunes separated. In order to achieve $\geq 50 \%$ beam polarization out of AGS, all of those spin resonances need to be corrected. This paper proposes three correction methods to overcome the strong intrinsic spin resonances as well as the weak intrinsic spin resonances and the coupling spin resonances.
\end{abstract}

\section{INTRODUCTION}

In the Brookhaven AGS, the polarized protons are accelerated from $G \gamma=4.5$ to $G \gamma=46.5$. A total of 42 imperfection spin resonances $[1,2]$ are crossed during the acceleration. A partial solenoid snake of $5 \%$ is used to avoid beam polarization loss at each imperfection resonance $[2,3]$.

Because of the AGS 12-fold symmetry, a total of 7 intrinsic spin resonances are harmful to the beam polarization. The intrinsic spin resonances are $G \gamma=$ $0+\nu_{z}, G \gamma=12+\nu_{z}, G \gamma=24-\nu_{z}, G \gamma=24+\nu_{z}, G \gamma=48-\nu_{z}, G \gamma=$ $36-\nu_{z}$, and $G \gamma=36+\nu_{z}$ where $\nu_{z}$ is the vertical betatron tune. Among them, the intrinsic resonances at $G \gamma=0+\nu_{z}, G \gamma=12+\nu_{z}$ and $G \gamma=36 \pm \nu_{z}$ are much stronger than the other three resonances. With the nominal AGS machine and beam conditions, i.e. $10 \pi \mathrm{mm}$-mrad normalized vertical beam emittance and $\alpha=4.8 \times 10^{-5}$ acceleration rate, the strong intrinsic resonances can cause total depolarization and even partial spin flips, while the weak spin resonances cause

1) This paper is a summary of M. Bai's three lectures at the Workshop. Work performed under the quspices of the US Department of Energy 
about 5 to $10 \%$ polarization losses. Currently, a vertical rf dipole is employed to induce a full spin flip at each strong intrinsic resonance without losing beam polarization [8]. For the weak spin resonances, no correction is implemented during acceleration.

With the normal AGS polarized proton setup, the horizontal and vertical betatron oscillations are coupled due to the solenoidal field of the $5 \%$ snake. Beam depolarization also occurs at the coupling spin resonances $G \gamma=k P \pm \nu_{x}$ where $k$ is an integer, $P=12$ is the super-periodicity of the AGS and $\nu_{x}$ is the horizontal betatron tune. The strength of the coupling resonance is proportional to the associated intrinsic resonance as well as the coupling strength. Currently, no correction schemes are applied at these resonances except separating the horizontal and vertical tunes.

In order to achieve more than $50 \%$ beam polarization at the AGS extraction energy, one needs to not only fully correct the imperfection spin resonances and the strong intrinsic spin resonances, but also address the beam depolarization at the weak intrinsic resonances and the coupling spin resonances.

\section{OVERCOMING STRONG INTRINSIC SPIN RESONANCES WITH AN RF DIPOLE}

The intrinsic spin resonance is driven by the quadrupole focusing magnetic fields due to the vertical betatron oscillation. The strength of an intrinsic spin resonance is proportional to the betatron oscillation amplitude. Normally in a beam, particles close to the core of the beam oscillate less than particles around the edge. Thus, the final polarization is an ensemble average of the Froissart-Stora formula over the betatron amplitude of the beam particles. Using the Gaussian beam distribution model, the final polarization becomes

$$
P_{f}=\left(\frac{1-\pi\left|\epsilon_{\mathrm{rms}}\right|^{2} / \alpha}{1+\pi\left|\epsilon_{\mathrm{rms}}\right|^{2} / \alpha}\right) P_{i}
$$

where $P_{i, f}$ is the beam polarization before/after crossing the spin resonance. $\epsilon_{\mathrm{rms}}$ is the spin resonance strength for a particle with an rms emittance. $\alpha$ is resonance crossing rate given by

$$
\alpha=\frac{d\left(G \gamma-k P \mp m \nu_{z}\right)}{d \theta}
$$

and $\theta$ is the orbiting angle in the synchrotron. For a given intrinsic spin resonance, no polarization will be lost if the resonance is crossed very fast, i.e. $\frac{\pi\left|\epsilon_{\mathrm{rms}}\right|^{2}}{\alpha} \ll 1$.

In general, there are alternatives to preserve the beam polarization through a spin resonance. One can either "jump" through the resonance so that $\pi\left|\epsilon_{\text {rms }}\right|^{2} / \alpha$ is close to zero; or slowly cross the spin resonance so that $\pi\left|\epsilon_{\mathrm{rms}}\right|^{2} / \alpha$ is close to infinity to achieve a spin flip. However, for a strong spin resonance, these two techniques 
are all limited because either the required amount of tune jump and speed of tune jump are not feasible or a very slow ramp rate would cause additional polarized losses from neighboring weak intrinsic spin resonances.

Alternatively, a full spin flip can also be obtained under the normal acceleration rate by enhancing the resonance strength. For intrinsic spin resonances, the effective resonance strength in Eq. (1) can be greatly enhanced in the presence of a large amplitude coherent oscillation, which can be adiabatically excited by an RF dipole. Since the betatron coordinate can be expressed as the linear combination of the vertical betatron motion and the coherent betatron motion [5], the particles experience not only the intrinsic spin resonance, but also a coherent spin resonance at the driving frequency. The resulting polarization, in the limiting case that the driving frequency coincides with the free oscillation frequency, is given by [6]

$$
\left\langle\frac{P_{f}}{P_{i}}\right\rangle=\frac{2}{1+\pi\left|\epsilon_{\mathrm{rms}}\right|^{2} / \alpha} \exp \left\{-\frac{\left(Z_{\mathrm{coh}}^{2} \hat{\beta}_{z} / 2 \beta_{z} \sigma_{z}^{2}\right)\left(\pi\left|\epsilon_{\mathrm{rms}}\right|^{2} / \alpha\right)}{1+\pi\left|\epsilon_{\mathrm{rms}}\right|^{2} / \alpha}\right\}-1,
$$

and in the case that the two resonances are well separated, by

$$
\left\langle\frac{P_{f}}{P_{i}}\right\rangle=\frac{1-\pi\left|\epsilon_{\mathrm{rms}}\right|^{2} / \alpha}{1+\pi\left|\epsilon_{\mathrm{rms}}\right|^{2} / \alpha}\left(2 \exp \left\{-\frac{Z_{\mathrm{coh}}^{2} \hat{\beta}_{z}}{\beta_{z} \sigma_{z}^{2}} \frac{\pi\left|\epsilon_{\mathrm{rms}}\right|^{2}}{2 \alpha}\right\}-1\right) .
$$

Here $Z_{c o h}$ is the coherent oscillation amplitude, $\hat{\beta}_{z}$ is the maximum vertical betatron function in the accelerator and $\sigma_{z}$ is the rms beam size. Any case in between can produce rich interference patterns and the beam polarization is determined by both the relative strengths and phases of the two resonances [7].

This method has been implemented in the AGS since 1997. The experimental results demonstrated a full spin flip induced by a vertical rf dipole at each strong intrinsic spin resonance [8]. Figure 1 shows the measured polarization at three intrinsic resonances vs the coherent oscillation amplitude which is proportional to the RF dipole strength. The data at spin resonance $12+\nu_{z}$ (in the middle plot) demonstrates that the spin was fully flipped at large coherent oscillations where the measured polarization saturated. The same result is also indicated from the data at $0+\nu_{z}$ (in the bottom plot) and $36-\nu_{z}$ (in the top plot) with the smallest resonance proximity parameter $\delta$. The systematic error of the beam polarization was estimated to be $10 \%$, and the statistical error was about $\pm 3 \%$. The lines shown in the figure correspond to results obtained from numerical spin simulations of two spin resonances model.

\section{OVERCOMING WEAK INTRINSIC SPIN RESONANCES WITH THE TUNE-JUMP METHOD}

For the weak intrinsic spin resonances, in order to achieve a full spin flip using the rf dipole, one needs to excite an extremely large vertical coherence. For the 


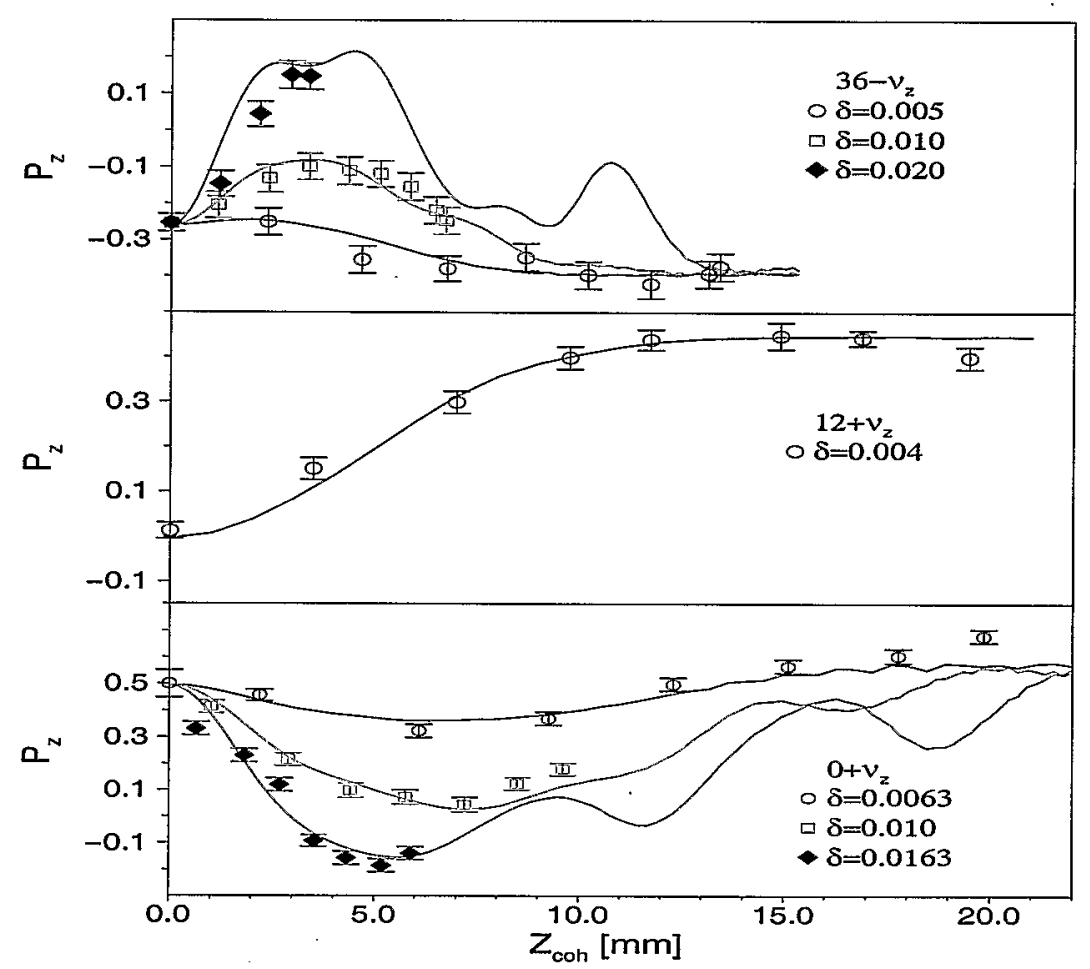

FIGURE 1. The measured proton polarization vs the coherent betatron oscillation amplitude (in $\mathrm{mm}$ ) for different tune separations at spin depolarizing resonances $0+\nu_{z}$ (bottom plot), $12+\nu_{z}$ (middle plot), and $36-\nu_{z}$ (upper plot) respectively. The error bars show only the statistical errors. The resonance strength of the coherent spin resonance due to the RF dipole is proportional to the coherent betatron amplitude. The lines are the results of multi-particle spin simulations based on the two nearby spin resonances model.

AGS, about $30 \sigma_{z}$ oscillation amplitude is required to induce a full spin flip at $G \gamma=48-\nu_{z}$ where the rms beam size $\sigma_{z}$ is $1.4 \mathrm{~mm}$ for a beam with vertical emittance of $10 \pi \mathrm{mm}-\mathrm{mrad}$. This certainly exceeds the limit of the AGS physical aperture $\left(6^{\prime \prime}(H) \times 3^{\prime \prime}(V)\right)$. However compared with strong intrinsic resonances, the much weaker strength allows one to be able to preserve the beam polarization with a moderate tune jump through the weak intrinsic resonances.

The principle of the tune-jump technique is to speed up the resonance crossing rate by pulsing a family of quadrupoles within a very short time. The schematic drawing in Fig. 2 shows how the tune-jump method works. The effective resonance crossing rate $\alpha_{t j}$ become

$$
\alpha_{t j}=\alpha+\frac{\left|\Delta \nu_{z}\right|}{\Delta \theta}
$$

where $\alpha$ is the original resonance crossing rate due to acceleration, $\Delta \nu_{z}$ is the total amount of tune-jump and $\Delta \theta$ is the tune-jump duration. The polarization of a 


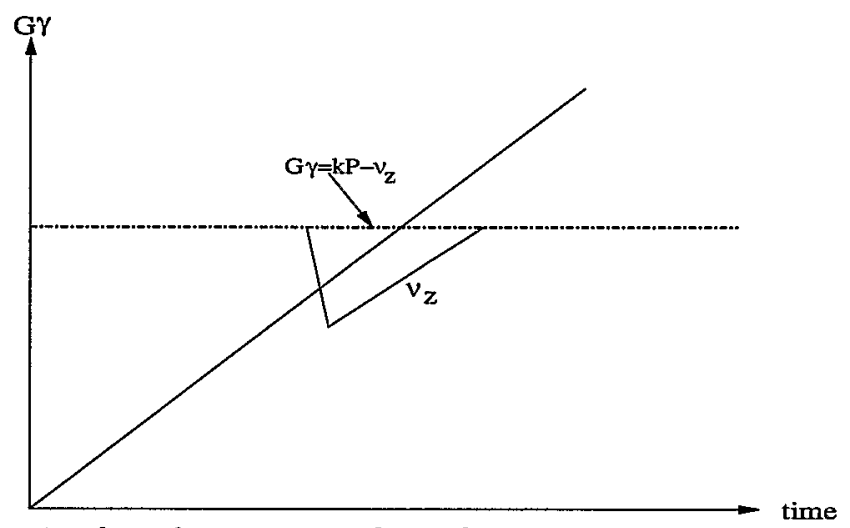

FIGURE 2. Schematic plot of tune-jump through a intrinsic resonance at $G \gamma=k P-\nu_{z}$. For the intrinsic resonance at $G \gamma=k P+\nu_{z}$, the tune jump will have the opposite polarity.

Gaussian distributed beam after tune-jumping through an intrinsic spin resonance is given by Eq. 1 .

In order to preserve the beam polarization through a weak intrinsic resonance, the size of the tune jump $\Delta \nu_{z}$ and the duration of the tune jump $\Delta \theta$ are the two critical parameters. Normally, the size of the tune jump is proportional to the strength of the resonance and the duration of the jump is determined by the desired resonance crossing rate based on Eq. 1. Table 1 lists the three weak spin resonances' strength for a beam with vertical $10 \pi$ mm-mrad normalized emittance as well as the tune-jump size and duration. Here, the tune-jump speed for the weak

TABLE 1. AGS weak intrinsic spin resonance strengths

\begin{tabular}{|c|c|c|c|c|c|}
\hline $\mathrm{P}[\mathrm{GeV} / \mathrm{c}]$ & $G \gamma$ & $\epsilon_{k}$ & $\Delta \nu_{z}$ & jump time & $\frac{p_{f}}{p_{i}}$ \\
\hline 7.95 & $24-\nu_{z}$ & 0.0002 & 0.11 & $1.89 \mathrm{~ms}$ & 0.99 \\
\hline 17.08 & $24+\nu_{z}$ & 0.0004 & 0.11 & $473 \mu \mathrm{s}$ & 0.99 \\
\hline 20.54 & $48-\nu_{z}$ & 0.0006 & 0.11 & $210 \mu s$ & 0.99 \\
\hline
\end{tabular}

intrinsic resonance at $G \gamma=24-\nu_{z}$ is less than the AGS nominal acceleration rate with Siemens power bank and no correction needs to be done for this resonance during the AGS normal operation.

This technique has been successfully demonstrated during the 1986 AGS polarized proton acceleration experiment [9]. A total of ten ferrite quadrupoles were pulsed at all the intrinsic spin resonances between the AGS injection energy of $200 \mathrm{MeV}$ and extraction energy of $22 \mathrm{GeV}$, except for $G \gamma=24-\nu_{z}$. Each pulsed quadrupole is $50 \mathrm{~cm}$ long and is capable of reaching a maximum field gradient of $11.7 \mathrm{kG} / \mathrm{m}$. The fast quadrupoles were designed to have a rise time of $1.6 \mu \mathrm{s}$. In order to achieve such a fast time constant, ceramic beam pipes without any coating were used in these magnets. The elliptical aperture of the ceramic pipe is $8.5 \mathrm{~cm}(\mathrm{H}) \times 7.4 \mathrm{~cm}(\mathrm{~V})$. During the experiment, the maximum achieved tune jump 
was 0.28 in $1.6 \mu s$.

Although this method was used to overcome the strong resonances at $G \gamma=0+\nu_{z}$, $G \gamma=12+\nu_{z}$ and $G \gamma=36-\nu_{z}$ during the experiment, the large tune-jump size (0.28) and incredible speed at strong spin resonance not only brought a great difficulty in system implementation but also caused beam emittance growth. The ceramic beam pipes later on became a problem for the AGS high intensity proton operation because of their high impedance and vertical aperture restrictions, and all the fast quadrupoles were later removed from the beam line.

Since the required tune-jump size and speed is significantly less for the weak spin resonances, this method is more suitable for crossing weak spin resonances. The moderate tune-jump size allows one to be able to afford a fast quadrupole with larger aperture. The much slower crossing speed also allows one to put in aluminum strips through the ceramic beam pipe to reduce the impedance for the AGS high intensity operation. For the AGS, $G \gamma=48-\nu_{z}$ is the strongest among the three weak intrinsic spin resonances. To achieve the tune-jump size listed in the Table 1, the desired quadrupole gradient is $5.5 \mathrm{kG} / \mathrm{m}$ assuming 10 fast quadrupoles distributed at the maximum vertical beta function $(25 \mathrm{~m})$ around the ring. Although to obtain a tune-jump time of $210 \mu s$, a ceramic beam pipe is inevitable, a set of aluminum strips can be placed outside the beam pipe to provide a path for the image current and thus reduce the impedance.

\section{COUPLING SPIN RESONANCES}

In a perfect accelerator, the horizontal and vertical betatron oscillations are independent of each other. However, this independence can be broken if there is any quadrupole roll error or solenoid field. In this case, the horizontal motion then gets coupled to the vertical oscillation. Unlike the uncoupled case, the frequency spectrum of the betatron oscillation in either of the two transverse plane then consists of two components $\nu_{1}$ and $\nu_{2}$ given by $[4,10]$

$$
\begin{aligned}
& \nu_{1}=\frac{1}{2}\left(\nu_{x}+\nu_{z}\right)+\frac{1}{2} \sqrt{\left(\nu_{x}-\nu_{z}\right)^{2}+\Delta Q_{\min }^{2}} \rightarrow \nu_{x} ; \text { without coupling } \\
& \nu_{2}=\frac{1}{2}\left(\nu_{x}+\nu_{z}\right)-\frac{1}{2} \sqrt{\left(\nu_{x}-\nu_{z}\right)^{2}+\Delta Q_{\min }^{2}} \rightarrow \nu_{z} ; \text { without coupling }
\end{aligned}
$$

where $\nu_{x}$ and $\nu_{z}$ are the unperturbed horizontal and vertical tune. $\Delta Q_{\min }$ is the minimum tune split between the two eigen tunes when $\nu_{x}=\nu_{z}$. It is given by

$$
\Delta Q_{\text {min }}=\frac{1}{2 \pi} \oint \sqrt{\beta_{x} \beta_{z}} A_{x z} e^{\left.i\left(\nu_{x} \phi_{x}-\nu_{z} \phi_{z}-\left(\nu_{x}-\nu_{z}-l\right) \frac{s}{R}\right)\right)} d s,
$$

where $\beta_{x, z}$ are the betatron amplitude functions for the horizontal and vertical planes and $A_{x z}$ is proportional to the strength of the coupling elements [11]. For a solenoid magnet, 


$$
A_{x z}=\frac{B / /}{2 B \rho}\left[\left(\frac{\alpha_{x}}{\beta_{x}}-\frac{\alpha_{z}}{\beta_{z}}\right)+i\left(\frac{1}{\beta_{x}}+\frac{1}{\beta_{z}}\right)\right]
$$

where $B_{/ /}$is the solenoid magnetic field strength and $B \rho$ is the momentum per charge. With weak coupling,

$$
\begin{aligned}
& \nu_{1} \simeq \nu_{x} \\
& \nu_{2} \simeq \nu_{z} .
\end{aligned}
$$

In the Brookhaven AGS, the main coupling source comes from the solenoid partial snake which is used to overcome the imperfection spin resonances in the AGS [2]. The snake is $2.4384 \mathrm{~m}$ long and located in the $I 10$ straight section where $\beta_{x}=$ $12.2 \mathrm{~m}, \beta_{z}=18.2 \mathrm{~m}, \alpha_{x}=1.050$ and $\alpha_{z}=-1.470$. For a $5 \%$ snake, $\frac{B_{l /}}{B \rho}=$ $0.023 \mathrm{~m}^{-1}$, and the minimum tune split $\Delta Q_{\min }$ from a $5 \%$ partial snake is

$$
\Delta Q_{\min }=0.01435 \text {. }
$$

In a coupled machine, in addition to the intrinsic spin resonance at $G \gamma=k P \pm$

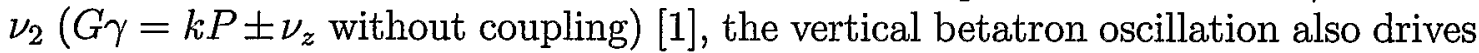
a coupling spin resonances at $G \gamma=k P \pm \nu_{1}$. The strength of the coupling resonance $\epsilon_{\nu_{x}}$ is proportional to the amount of the coupling and is given by

$$
\epsilon_{\nu_{x}} \propto C_{x} \sqrt{\varepsilon_{u}} \epsilon_{\nu_{z}}
$$

where $\epsilon_{\nu_{z}}$ is the strength of the adjacent intrinsic spin resonance and $C_{x}$ is the coupling coefficient. For a fully coupled machine, $\nu_{x}=\nu_{z}$ and $C_{x}=1$. For a decoupled machine, $C_{x}=0 . \varepsilon_{u}$ is the beam emittance in the eigen direction [12] and equals the horizontal beam emittance if $C_{x}=0$.

Traditionally in the AGS, the beam polarization loss at the coupling resonances is minimized by separating the horizontal and vertical set points. The coupling resonances around these four strong intrinsic spin resonances can produce about $35 \%$ polarization losses with the normal AGS polarized proton setting [3,13]. In order to achieve $70 \%$ polarization in the AGS, one needs to minimize the polarization loss at the coupling resonances. Because they are adjacent to the intrinsic resonances, it is very difficult to use the vertical rf dipole [8] to obtain two full spin flips at both the intrinsic and the coupling resonances.

\section{A Using a Horizontal rf Dipole to Cross the Intrinsic and Coupling resonances}

Analogous to the method of using a vertical rf dipole at the intrinsic spin resonance, one should also expect to obtain a full spin flip by inducing a strong artificial resonance if the intrinsic and its coupling spin resonances are fully overlapped. Because of the coupling effect, the two spin resonance can never be brought closer 
than the minimum tune split $\Delta Q_{\min }$. However, $\Delta Q_{\min }$ in general is small and a full spin-flip still should be achievable if the induced resonance is strong enough. In a fully coupled machine, the unperturbed tunes are equal and the intrinsic and the coupling resonances are equally strong and located on either side of the unperturbed betatron tune at a distance of half of $\Delta Q_{\min }$.

Unlike using a vertical rf dipole to obtain a vertical coherence in an uncoupled machine [5], the vertical coherence is excited by a horizontal $\mathrm{rf}$ dipole in a fully coupled machine. To understand this, let us first transform the beam motion from the normal geometric coordinates $(x, z, s)$ to the $(u, v, s)$ coordinate system in which the betatron motions along the two eigen directions are fully decoupled [12], i.e.

$$
\left(\begin{array}{c}
x \\
x^{\prime} \\
z \\
z^{\prime}
\end{array}\right)=R\left(\begin{array}{c}
u \\
u^{\prime} \\
v \\
v^{\prime}
\end{array}\right)
$$

where $R$ is the transformation matrix between $(x, z, s)$ and $(u, v, s)$. In a fully coupled machine with weak coupling coming from a solenoid magnet, the minimum tune split $\Delta Q_{\min }<<1$ and the $R$ matrix is given by (See the Appendix)

$$
R=\frac{1}{\sqrt{2}}\left(\begin{array}{cccc}
1 & 0 & -a\left(\alpha_{x}+\alpha_{z}\right) & -a\left(\beta_{x}+\beta_{z}\right) \\
0 & 1 & a\left(\gamma_{x}+\gamma_{z}\right) & a\left(\alpha_{x}+\alpha_{z}\right) \\
-a\left(\alpha_{x}+\alpha_{z}\right) & -a\left(\beta_{x}+\beta_{z}\right) & 1 & 0 \\
a\left(\gamma_{x}+\gamma_{z}\right) & a\left(\alpha_{x}+\alpha_{z}\right) & 0 & 1
\end{array}\right)
$$

where $a=\frac{1}{\sqrt{\beta_{x} \gamma_{z}+\beta_{z} \gamma_{x}+2\left(1-\alpha_{x} \alpha_{z}\right)}}$ and $\beta_{x, z}$ are the horizontal and vertical betatron functions at the position of the solenoid magnet. $\gamma_{x, z}=\frac{1+\alpha_{x, z}^{2}}{\beta_{x, z}}$ and $\alpha_{x, z}=-\frac{1}{2} \beta_{x, z}^{\prime}$ are the corresponding twiss parameters. Here ${ }^{\prime}$ is the derivative with respect to the longitudinal coordinate $s$. With the weak coupling force, one also has [14]

$$
\begin{gathered}
\beta_{u} \simeq \beta_{x} ; \quad \beta_{v} \simeq \beta_{z} \\
\alpha_{u} \simeq \alpha_{x} ; \quad \alpha_{v} \simeq \alpha_{z}
\end{gathered}
$$

where $\beta_{u, v}$ and $\alpha_{u, v}$ are the Courant-Snyder parameters in the two eigen directions.

With a horizontal rf dipole $\Delta B_{y} L=\Delta B_{y m} L \cos \nu_{m} \theta$, the horizontal excitation is $\delta x^{\prime} \cos \nu_{m} \theta=\frac{\Delta B_{y_{m} L} L}{B \rho} \cos \nu_{m} \theta$, where $B \rho$ is the magnetic rigidity. The corresponding excitation in the $(u, v)$ coordinates are

$$
\begin{array}{r}
\delta u=0 \\
\delta u^{\prime}=\frac{1}{\sqrt{2}} \delta x^{\prime} \\
\delta v=\frac{a}{\sqrt{2}}\left(\beta_{x}+\beta_{z}\right) \dot{\delta x^{\prime}} \\
\delta v^{\prime}=-\frac{a}{\sqrt{2}}\left(\alpha_{x}+\alpha_{z}\right) \delta x^{\prime} .
\end{array}
$$


Similar to the case of using a vertical rf dipole in an uncoupled machine [5], the coherent betatron motion is a fixed point in the frame which rotates with the rf dipole modulation frequency as shown in Fig. 3. During every modulation period,

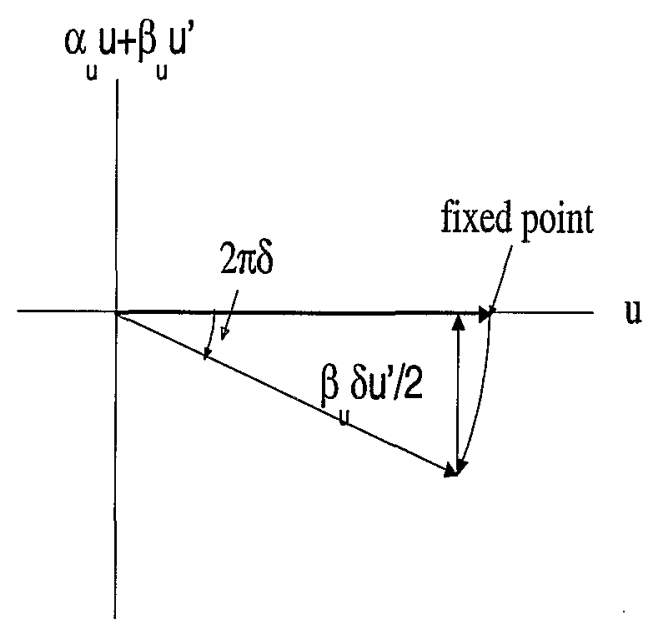

FIGURE 3. Derivation of the coherent betatron motion in the $\left(u, u^{\prime}\right)$ plane in the rotating frame. In each modulation period, the phase-space vector rotates through an angle of $2 \pi \delta$ and is then given an effective kick of $\frac{\beta_{u} \delta u^{\prime}}{2}$ by the rf dipole field.

the particle's phase-space vector rotates through an angle of $2 \pi \delta$ in the rotating frame where $\delta=\left|\nu_{m}-\nu_{1,2}\right|$. It then gets deflected by the rf dipole. The effective deflection is $\frac{1}{2} \beta_{u} \delta u^{\prime}$ due to the fact that the deflection given by the rf dipole is oscillating at the same frequency. Hence, the fixed point in the $\left(u, u^{\prime}\right)$ plane is at

$$
\begin{array}{r}
f_{u}=b \frac{a}{\sqrt{2}} \beta_{x} \delta x^{\prime} \\
f_{u^{\prime}}=-\frac{\alpha_{x}}{\beta_{x}} b \frac{a}{\sqrt{2}} \beta_{x} \delta x^{\prime}
\end{array}
$$

where $b=\frac{1}{4 \pi \delta}$. The fixed point in the $\left(v, v^{\prime}\right)$ plane can be calculated using the same method. Since $\delta v \neq 0$, the deflection given by the rf dipole in the $\left(v, v^{\prime}\right)$ plane is not along the axis of $\alpha_{v} v+\beta_{v} v^{\prime}$. The fixed point in the $\left(v, v^{\prime}\right)$ plane is given by

$$
\begin{aligned}
& f_{v}=-b \frac{a}{\sqrt{2}}\left(\alpha_{z} \beta_{x}-\beta_{z} \alpha_{x}\right) \delta x^{\prime} \\
& f_{v^{\prime}}=b \frac{a}{\sqrt{2}}\left(\beta_{x} \gamma_{z}-\alpha_{x} \alpha_{z}+1\right) .
\end{aligned}
$$

Transforming the fixed points in the $(u, v)$ coordinate system back to the normal geometric $(x, z)$ coordinates,

$$
f_{x} / b=\frac{1}{\sqrt{2}} f_{u}-\frac{a}{\sqrt{2}}\left(\alpha_{x}+\alpha_{z}\right) f_{v}-\frac{a}{\sqrt{2}}\left(\beta_{x}+\beta_{z}\right) f_{v^{\prime}}
$$




$$
\begin{aligned}
f_{x^{\prime}} / b & =\frac{1}{\sqrt{2}} f_{u^{\prime}}+\frac{a}{\sqrt{2}}\left(\gamma_{x}+\gamma_{z}\right) f_{v}+\frac{a}{\sqrt{2}}\left(\alpha_{x}+\alpha_{z}\right) f_{v^{\prime}} \\
f_{z} / b & =-\frac{a}{\sqrt{2}}\left(\alpha_{x}+\alpha_{z}\right) f_{u}-\frac{a}{\sqrt{2}}\left(\beta_{x}+\beta_{z}\right) f_{u^{\prime}}+\frac{1}{\sqrt{2}} f_{v} \\
f_{z^{\prime}} / b & =\frac{a}{\sqrt{2}}\left(\gamma_{x}+\gamma_{z}\right) f_{u}+\frac{a}{\sqrt{2}}\left(\alpha_{x}+\alpha_{z}\right) f_{u^{\prime}}+\frac{1}{\sqrt{2}} f_{v^{\prime}}
\end{aligned}
$$

By substituting Eq. 25 to Eq. 26, one then obtains

$$
\begin{array}{r}
f_{x} / b=0 \\
f_{x^{\prime}} / b=0 \\
f_{z} / b=a\left(\beta_{z} \alpha_{x}-\beta_{x} \alpha_{z}\right) \delta x^{\prime} \\
f_{z^{\prime}} / b=a\left(\beta_{x} \gamma z-\alpha_{x} \alpha_{z}+1\right) \delta x^{\prime} .
\end{array}
$$

This demonstrates that in a fully coupled machine, a vertical coherence can be excited by applying a horizontal rf dipole. The amplitude of the vertical coherence is $\frac{B L}{4 \pi B \rho \delta} \sqrt{\beta_{x} \beta_{z}}$.

Fig. 4 shows the numerical spin tracking results at $G \gamma=36+\nu_{z}$. The dotted line shows the result with the nominal AGS tune setting $\left(\nu_{x}=8.8, \nu_{z}=8.7\right)$ and no correction scheme for the intrinsic spin resonance. In this case, the depolarization at the coupling resonance is obvious. The solid line is the result of using a horizontal rf dipole with the horizontal and vertical betatron tunes set at 8.7. Due to the coupling from the solenoid partial snake, the two betatron tunes are split by 0.0144 . The horizontal rf dipole tune was set at 0.3. With a horizontal RF dipole amplitude 28.0 G-m, a full spin flip was achieved.

\section{B Experimental results}

The method of using a horizontal rf dipole to excite a vertical coherence to cross the coupling spin resonance was tested in the AGS during the 2000 RHIC polarized proton commissioning run. The polarized $H^{-}$beam was pre-accelerated in the $200 \mathrm{MeV}$ LINAC and then stripped and injected into the Booster. It was then injected into the AGS at $G \gamma=4.7$ and accelerated up to $G \gamma=46.5$. In the AGS, the nominal tune setting is $\nu_{x}=8.8$ and $\nu_{z}=8.7$.

During the experiment, the AGS skew quadrupoles were all set to $17 \mathrm{~A}$. Due to a hardware limit, the partial snake strength at $G \gamma=36+\nu_{z}$ was actually only about $3.5 \%$ instead of $5 \%$. The combined effect of the skew quadrupoles and the weaker snake gave a smaller minimum tune spilt $\Delta Q_{\min }$ of 0.007 . The horizontal rf dipole was set in the middle of the two betatron tunes $\nu_{1}$ and $\nu_{2}$. The turn-byturn beam position monitor data confirmed that a vertical coherence was excited without horizontal response as shown in the left part of Fig. 5. The horizontal response was not zero once the rf dipole tune deviated from the average of the two eigen tunes as shown on the right of Fig. 5. 


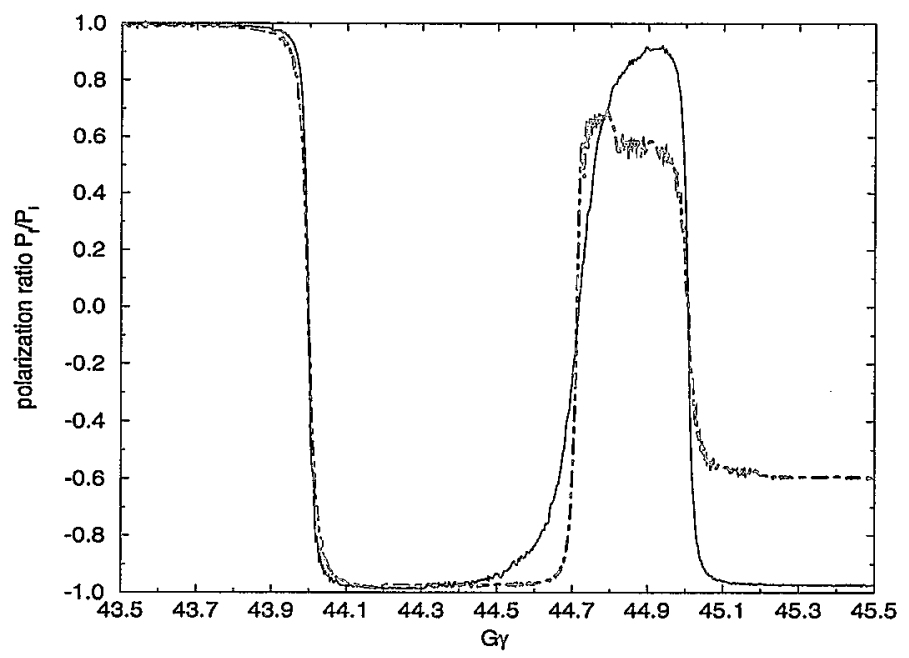

FIGURE 4. This figure shows the calculated polarization ratio $P_{f} / P_{i}$ as a function of energy. The solid line is for the case of a fully coupled machine and a horizontal rf dipole used to obtain an adiabatic vertical coherence. The dotted line is the result of a weakly coupled machine with the two betatron tunes set 0.1 apart. No correction scheme was used at the $G \gamma=36+\nu_{z}$ intrinsic spin resonance. For both cases, the horizontal and vertical emittance are $20 \pi \mathrm{mm}-\mathrm{mrad}$ and $10 \pi \mathrm{mm}$-mrad respectively.

Table 2 shows the comparison of the measured beam asymmetries of using vertical rf dipole, no correction and using horizontal rf dipole at $G \gamma=36+\nu_{z}$. Comparing the measured asymmetry when using the horizontal rf dipole with the case of no correction, it is clear that the horizontal rf dipole did help to recover the beam polarization. However, the excited coherence was not optimized and about $70 \%$

TABLE 2. Measured beam asymmetries

\begin{tabular}{|c|c|c|}
\hline & Measured asymmetry $\left(\mathrm{x} 10^{-3}\right)$ & Condition \\
\hline 1 & $1.50 \pm 0.04$ & with vertical rf dipole \\
\hline 2 & $1.25 \pm 0.1$ & with horizontal rf dipole \\
\hline 3 & $0.067 \pm 0.063$ & no correction \\
\hline
\end{tabular}

beam emittance growth was observed. Because of limitations of the AGS sextupole power supplies, we could not achieve small chromaticities in both planes and obtain a fully adiabatic excitation. This was the most likely reason that the horizontal rf dipole did not recover $100 \%$ beam polarization as expected. 


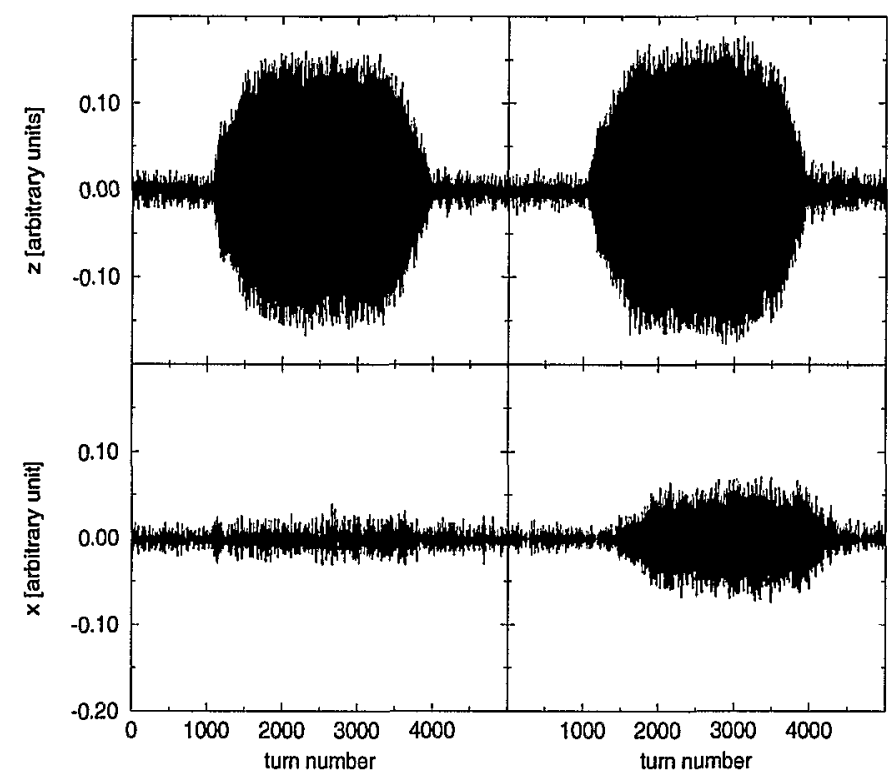

FIGURE 5. The top and bottom plots on the left are vertical and horizontal turn-by-turn beam position data when the horizontal rf dipole modulation tune $\nu_{m}=\frac{1}{2}\left(\nu_{1}+\nu_{2}\right)$. As shown, no horizontal coherence was excited. The two plots on the right correspond to the case where the horizontal modulation tune $\nu_{m} \neq \frac{1}{2}\left(\nu_{1}+\nu_{2}\right)$ and the horizontal coherence was no longer zero.

\section{ACKNOWLEDGEMENT}

The three techniques discussed in this report were done in the AGS over several years and several papers have been published in various journals as well as conference proceedings. The authors would like to thank all the people who have been involved in these research. In addition, we would also like to thank Dr. E. D. Courant, W. J. Glenn, Dr. H. Huang, Dr. A. Lehrach, Dr. A. Luccio, Dr. W. Mackay, V. Ranjbar, Dr. N. Tsoupas, Dr. W. van Asselt for the fruitful discussions. We also would like to thank K. Zeno and D. Warburton for their great help. This work is performed under the auspices of Department of Energy of U.S.A.

\section{APPENDIX}

In an accelerator with a solenoid magnet as the only source of coupling, the one-turn matrix $T$ is

$$
T=\left(\begin{array}{cc}
M & n \\
m & N
\end{array}\right)=\left(\begin{array}{cc}
p & q \\
-q & p
\end{array}\right)\left(\begin{array}{cc}
M_{1} & 0 \\
0 & N_{1}
\end{array}\right)
$$

where $M_{1}$ and $N_{1}$ are the matrices starting from the beginning of the solenoid to the end of the solenoid. $M$ and $N$ are the horizontal and vertical matrices. 


$$
M=\left(\begin{array}{cc}
\cos \mu_{x}+\alpha_{x} \sin \mu_{x} & \beta_{x} \sin \mu_{x} \\
-\gamma_{x} \sin \mu_{x} & \cos \mu_{x}-\alpha_{x} \sin \mu_{x}
\end{array}\right)
$$

and

$$
N=\left(\begin{array}{cc}
\cos \mu_{z}+\alpha_{z} \sin \mu_{z} & \beta_{z} \sin \mu_{z} \\
-\gamma_{z} \sin \mu_{z} & \cos \mu_{z}-\alpha_{z} \sin \mu_{z}
\end{array}\right)
$$

where $\alpha_{x, z}, \beta_{x, z}$ and $\gamma_{x, z}$ are the horizontal and vertical Courant-Snyder parameters. $\mu_{x, z}$ are the horizontal and vertical phase advances. $p$ and $q$ are the components in the solenoid transfer matrix $T_{s}$.

$$
T_{s}=\left(\begin{array}{cc}
p & q \\
-q & p
\end{array}\right)=\left(\begin{array}{cccc}
C^{2} & \frac{S C}{K} & S C & \frac{S^{2}}{K} \\
-K S C & C^{2} & -k S^{2} & S C \\
-S C & -\frac{S^{2}}{K} & C^{2} & \frac{S C}{K} \\
k S^{2} & -S C & -K S C & C^{2}
\end{array}\right)
$$

where $K=\frac{B_{/ l}}{2 B \rho}, C=\cos (K L), S=\sin (K L)$ and $L$ is the length of the solenoid. $B / /$ is the strength of the solenoid. From Eq. 28, one has

$$
\begin{array}{r}
p M_{1}=M ; \\
p N_{1}=N ; \\
n=q N_{1}=q p^{-1} N ; \\
m=-q M_{1}=q p^{-1} M ;
\end{array}
$$

In a fully coupled machine where $\mu_{x}=\mu_{z}$, the transformation matrix $R$ which diagonalizes the one turn matrix $T$ in Eq. 28 is given by [2]

$$
R=\left(\begin{array}{cc}
\sqrt{1-|E|} & -s E s \\
-E & \sqrt{1-|E|}
\end{array}\right)
$$

where

$$
s=\left(\begin{array}{cccc}
0 & 1 & 0 & 0 \\
-1 & 0 & 0 & 0 \\
0 & 0 & 0 & 1 \\
0 & 0 & -1 & 0
\end{array}\right)
$$

and

$$
E=-\frac{\sqrt{2}}{4 \sin \mu \sin \left(\pi \Delta Q_{\min }\right)}(m-s n s) .
$$

where $\Delta Q_{\min }$ is the minimum tune split given by [11] 


$$
\Delta Q_{\min }=\frac{K L}{2 \pi} \sqrt{\beta_{x} \beta_{z}}\left[\left(\frac{\alpha_{x}}{\beta_{x}}-\frac{\alpha_{z}}{\operatorname{bet} a_{z}}\right)+i\left(\frac{1}{\beta_{x}}+\frac{1}{\beta_{z}}\right)\right]
$$

where $K L=\frac{B / / L}{2 B \rho}$. The size of the minimum tune split is

$$
\Delta Q_{\min }=\frac{K L}{2 \pi} a=\frac{K L}{2 \pi} \sqrt{\beta_{x} \gamma_{z}+\beta_{z} \gamma_{x}+2\left(1-\alpha_{x} \alpha_{z}\right)} .
$$

In a fully coupled machine with weak coupling strength, namely $\Delta Q_{\text {min }} \ll 1$ and $K L \ll 1$, we then have

$$
\sin \left(\pi \Delta Q_{\min }\right) \simeq \pi \Delta Q_{\min }
$$

and

$$
\frac{\sin (K L)}{\cos (K L)} \simeq K L
$$

Substituting Eq. 42, Eq. 43, $m$ in Eq. 36 and $n$ in Eq. 35 into Eq. 39, one then gets

$$
E=-\frac{1}{\sqrt{2}} a\left(\begin{array}{cc}
-\left(\alpha_{x}+\alpha_{z}\right) & -\left(\beta_{x}+\beta_{z}\right) \\
\left(\gamma_{x}+\gamma_{z}\right) & \left(\alpha_{x}+\alpha_{z}\right)
\end{array}\right)
$$

Hence, the $R$ matrix is

$$
R=\frac{1}{\sqrt{2}}\left(\begin{array}{cccc}
1 & 0 & -a\left(\alpha_{x}+\alpha_{z}\right) & -\left(\beta_{x}+\beta_{z}\right) \\
0 & 1 & \left(\gamma_{x}+\gamma_{z}\right) & \left(\alpha_{x}+\alpha_{z}\right) \\
-a\left(\alpha_{x}+\alpha_{z}\right) & -\left(\beta_{x}+\beta_{z}\right) & 1 & 0 \\
\left(\gamma_{x}+\gamma_{z}\right) & \left(\alpha_{x}+\alpha_{z}\right) & 0 & 1
\end{array}\right)
$$

and its inverse matrix $R^{-1}$ is

$$
R^{-1}=\frac{1}{\sqrt{2}}\left(\begin{array}{cccc}
1 & 0 & a\left(\alpha_{x}+\alpha_{z}\right) & \left(\beta_{x}+\beta_{z}\right) \\
0 & 1 & -\left(\gamma_{x}+\gamma_{z}\right) & -\left(\alpha_{x}+\alpha_{z}\right) \\
a\left(\alpha_{x}+\alpha_{z}\right) & \left(\beta_{x}+\beta_{z}\right) & 1 & 0 \\
-\left(\gamma_{x}+\gamma_{z}\right) & -\left(\alpha_{x}+\alpha_{z}\right) & 0 & 1
\end{array}\right)
$$

\section{REFERENCES}

1. E. D. Courant, R. D. Ruth, The Acceleration of Polarized Protons in Circular Accelerators, BNL report, BNL 51270, 1980.

2. T. Roser, Partial Siberian Snake Test at the Brookhaven AGS, in High Energy Spin Physics: 10th International Symposium, ed. T.Hasegawa, et al., Nagoya, Japan, 1992 (Universal Academic Press, Inc.,1992), p. 429.

3. H. Huang et al., Preservation of Proton Polarization by a Partial Siberian Snake, Phy. Rev. Letters. 73, 2982 (1994). 
4. S. Y. Lee, Accelerator Physics, World Scientific Pub. Singapore, 1999.

5. M. Bai, et al., Experimental Test of Coherent Betatron Resonance Excitations, Physical Review E, 5, p. 6002(1997).

6. M. Bai, S. Y. Lee, H. Huang, T. Roser and M. Syphers, AGS/RHIC/SN No. 055.

7. S. Tepikian, S. Y. Lee, E. D. Courant, Particle Accelerators 20, 1 (1986).

8. M. Bai et al., Overcoming Intrinsic Spin Resonances with an rf Dipole, Physical Review Letters 80, 4673(1998).

9. F. Z. Khiari et al., Acceleration of polarized protons to $22 \mathrm{GeV} / \mathrm{c}$ and the measurement of spin-spin effects in $p_{\uparrow}+p_{\uparrow} \rightarrow p+p$, Physical Review D, 39, p. 45(1989).

10. D. A. Edwards, M. J. Syphers, An Introduction To The Physics of High Every Accelerators, Wiley-Interscience Pub. 1993.

11. S. Y. Lee, Spin Dynamics and Snakes in Synchrotrons, World Scientific Pub. Singapore, 1997.

12. D. A. Edwards, L. C. Teng, Parametrization of LINEAR Coupled Motion in Periodic Systems, IEEE Trans. on Nucl. Sc. 20, 885 (1973).

13. H. Huang et al., Polarized Proton Beam in the AGS, Proceedings of $13^{\text {th }}$ international symposium in High Energy Spin Physics, p. 492 (1998).

14. T. Roser, Multiturn Injection With Coupling, AGS/AD/Tech. Note No. 354. 